\title{
НЕЛИНЕЙНЫЕ ЭФФЕКТЫ В РЕКОНФИГУРИРУЕМОЙ АНТЕННЕ
}

\author{
Д. С. ГАВВА, Е. А. МЕДВЕДЕВ, Е. А. ИВАНОВА, Е. В. ШАРАПОВА \\ Харьковский национальный университет радиоэлектроники, \\ Украина, Харьков, 61166, пр-т Науки, 14
}

\begin{abstract}
Аннотация. Современные телекоммуникационные системы, работающие с различными стандартами передачи данных, часто строятся на основе использования реконфигурируемых антенн. Включение в излучающую структуру антенны нелинейных радиоэлементов позволяет получить не только расширение функциональных возможностей антенны (переключение рабочей частоты, изменение формы диаграммы направленности, изменение поляризации, входного импеданса и т. п.), но и может вызывать ряд нелинейных эффектов, искажающих характеристики антенны и передаваемые данные. В связи с этим актуальными являются вопросы, рассмотренные в данной работе. А именно: описана методика численного анализа нелинейных эффектов в реконфигурируемых антеннах, рассмотрены особенности применяемых в антеннах нелинейных коммутационных радиоэлементов, проведены численные исследования влияния вольт-фарадной характеристики коммутатора излучающей структуры и мощности возбуждения на характеристики антенны. Выполненные оценочные исследования позволяют получать общие представления и осуществлять прогнозирование нелинейных искажений в реконфигурируемых антеннах, вызванных присутствием в них коммутаторов различных типов.
\end{abstract}

Ключевые слова: реконфигурируемая антенна; коммутация электродинамической структуры; МЭМС; вольт-фарадная характеристика; искажения сигнала

\section{1. ВВЕДЕНИЕ}

Развитие и улучшение характеристик современных радиоэлектронных устройств и систем сегодня в значительной мере идет по пути миниатюризации и расширения функциональных возможностей уже существующих радиоустройств. Достигается это за счет объединения электродинамических структур с нелинейными радиоэлементами (диоды, транзисторы, микроэлектромеханические структуры (МЭМС), и др.), а также реализации электродинамических устройств используя материалы, обладающие свойствами высокотемпературной сверхпроводимости.

Например, для реализации работы мобильного терминала связи с помощью различных стандартов передачи данных (Wi-Fi,
Bluetooth, 3G, 4G и т.д.) с использованием одной антенны используются реконфигурируемые антенны (РА). При этом имеется возможность не только перестраиваться по частоте, но также изменять положение главного лепестка диаграммы направленности (ДН), поляризацию, и другие характеристики.

К сожалению, присутствие в электродинамических структурах в целом и РА в частности, радиоэлементов с нелинейными характеристиками и/или материалов с нелинейными свойствами поверхностного импеданса дает не только положительные свойства. При определенных условиях и режимах работы и при определенных характеристиках «нелинейности» в радиоустройствах могут возникать различного рода нелинейные эффекты: интермодуляци- 


\section{БИБЛИОГРАФИЧЕСКИЙ СПИСОК}

1. Лучанинов, А.И.; Гавва, Д. С.; Шарапова, Е. В. Нелинейные эффекты в элементах электродинамических микрополосковых устройств на основе высокотемпературных сверхпроводников. Харьков: Коллегиум, 2015. 159 с. ISBN 978-966-97351-2-6.

2. Сидоров, Я. Г.; Гава, Д.С. "Нелинейные свойства реконфигурируемых антенн. Часть 1: Моделирование реконфигурируемой двухчастотной антенны," Восточно-Европейский журнал передовых техноло- 
гuй, T. 3, № 3, C. 52-56, 2009. URI: http://journals.uran. ua/eejet/article/view/20288.

3. Сидоров, Я. Г. “Математическая модель реконфигурируемых антенн с учетом нелинейных свойств управляющих элементов," Радиотехника (Харьков), № 155, С. 274-281, 2008.

4. Лучанинов, А.И.; Гавва, Д.С.; Крикун, Е.В.; Скорикова, Ю. В. “Структурный синтез реконфигурируемых антенн," Восточно-Европейский журнал передовых технологий, Т. 4, № 9, С. 28-34, 2010. URI: http://journals.uran.ua/eejet/article/view/3037.

5. Anagnostou, D. E. "Technological advances in reconfigurable and autonomous antenna systems," Proc. of 8th European Conf. on Antennas and Propagation, EuCAP 2014, 6-11 Apr., 2014, Hague, Netherlands. IEEE, 2014, p. 1727-1730. DOI: 10.1109/EuCAP.2014.69021 $\underline{25}$.

6. Jokic, I.; Frantlovic, M.; Z. Djuric. "RF MEMS and NEMS components and adsorption-desorption induced phase noise," Proc. of 29th Int. Conf. on Microelectronics, MIEL-2014, 12-14 May 2014, Belgrade, Serbia. IEEE, 2014, p. 117-124. DOI: $\underline{10.1109 /}$ MIEL.2014.6842100.

7. Attar, S. S.; Setoodeh, Sormeh; Mansour, Raafat R.; Gupta, Deepnarayan. "Low-temperature superconducting DC-contact RF MEMS switch for cryogenic reconfigurable RF front-ends," IEEE Trans. Microwave Theory Tech., Vol. 62, No. 7, P. 1437-1447, 2014. DOI: $10.1109 /$ TMTT.2014.2327205.

8. Кукушкин, А.Е.; Бичурин, М.И.; Татаренко, А.С.; Семенов, Г.А. "Применение МЭМС-технологий при разработке переменных СВЧ-аттенюаторов," Вестник Новгородского государственного универсиmema, № 6, C. 77-81, 2015. URI: http://www.novsu.ru/ vestnik/vestnik/i.78099/?article $=1202163$.

9. Dussopt, L.; Rebeiz, G. M. "Intermodulation distortion and power handling in RF MEMS switches, varactors, and tunable filters," IEEE Trans. Microwave Theory Tech., Vol. 51, No. 4, P. 1247-1256, 2003. DOI: 10.1109/TMTT.2003.809650.

10. Rebeiz, Gabriel M. RF MEMS Theory, Design, and Technology. New Jersey: John Wiley \& Sons, Inc., Hoboken, 2003. ISBN: 978-0-471-20169-4.

11. Гавва Д.С. “Представление поверхностного импеданса при моделировании электродинамических устройств выполненных на основе ВТСП материалов,” Радиотехника (Харьков), № 182, С. 121-129, 2015.

12. Лучанинов, А.И.; Гавва, Д.С.; Крикун, Е.В.; Вишнякова, Ю. В. "Результаты исследования нелинейных эффектов в микрополосковых линиях с ВТСП," Восточно-Европейский журнал передовых технологий, Т. 2, № 9, С. 4-8, 2012. URI: http://journals. uran.ua/eejet/article/view/3784.

13. Cho, S.; Lee, S. "Intermodulation measurements in superconducting meander lines," IEEE Trans. Applied Superconductivity, Vol. 9, No. 2, P. 3998-4001, 1999. DOI: $10.1109 / 77.783904$.
14. Vähä-Heikkilä, Tauno. MEMS tuning and matching circuits, and millimeter wave on-wafer measurements. Dissertation for the degree of Doctor of Science in Technology. VTT Technical Research Centre of Finland, 2006. ISBN 951-38-6705-6.

15 Arathy, U. S.; Resmi, R. "Analysis of pull-in voltage of MEMS switches based on material properties and structural parameters," Proc. of Int. Conf. on Control, Instrumentation, Communication and Computational Technologies, ICCICCT, 18-19 Dec. 2015, Kumaracoil, India. IEEE, 2015, P. 57-61. DOI: 10.1109/ICCICCT. 2015.7475249.

16. Bhatasana, Piyush; Pujara, Dhaval; Bera, S. C. "Movable parallel plate RF MEMS switch with wide frequency response," Proc. of Applied Electromagnetics Conf., AEMC, 18-21 Dec. 2015, Guwahati, India. IEEE, 2015, P. 1-2. DOI: 10.1109/AEMC.2015.7509162.

17. Girbau, David; Otegi, Nerea; Pradell, Lluís; Lázaro, Antonio. "Generation of third and higher-order intermodulation products in MEMS capacitors, and their effects," Proc. of 13th EGAAS Symp., 3-4 Oct. 2005, Paris, France. IEEE, 2005, P. 593-596. DOI: 10.1109/EUMC. 2005.1610245 .

18. Herfst, R. W.; Huizing, H. G. A.; Steeneken, P. G.; Schmitz, J. "Characterization of dielectric charging in RF MEMS capacitive switches," Proc. of IEEE Int. Conf. on Microelectronic Test Structures, 6-9 Mar. 2006, Austin, TX, USA. IEEE, 2006, P. 133-136. DOI: 10.1109/ ICMTS.2006.1614290.

19. Peroulis, D.; Pacheco, S.; Sarabandi, K.; Katehi, P. B. "MEMS devices for high isolation switching and tunable filtering," IEEE MTT-S Int. Microwave Symp. Dig., 11-16 Jun. 2000, Boston, MA, USA. IEEE, 2000, P. 1217-1220. DOI: 10.1109/MWSYM.2000.863578.

20. Shifrin, Y. S.; Luchaninov, A. I.; Gavva, D. S.; Zhurbenko, V. V. "Excitation of wire structures with nonlinear characteristics of the surface impedance," Proc. of 5th Int. Conf. on Antenna Theory and Techniques, 24-27 May 2005, Kyiv, Ukraine. IEEE, 2005, P. 156-159. DOI: 10.1109/ICATT.2005.1496909.

21. Gavva, D. S. "Characteristics of arbitrarily configurated thin wire antennas with the nonlinear surface impedance," Proc. of 4th Int. Conf. on Antenna Theory and Techniques, 9-12 Sept. 2003, Sevastopol, Ukraine. IEEE, 2003, P. 852-855. DOI: 10.1109/ICATT.2003.123 $\underline{8887}$.

22. Гавва, Д. С.; Лучанинов, А. И.; Омаров, М. А. "Характеристики проволочных электродинамических структур, возбуждаемых источниками различных типов,” Радиотехника (Харьков), № 134, С. 256-260, 2003.

23. Лучанинов, А. И.; Гавва, Д. С.; Уайд, С. Р. "Вибраторы с неравномерным распределением нелинейности поверхностного импеданса," Радиотехника (Харьков), № 177, С. 202-222, 2014.

24. Mansour, R. R.; Bakri-Kassem, M.; Daneshmand, M.; Messiha, N. "RF MEMS devices," Proc. of Int. Conf. on MEMS, NANO and Smart Systems, ICMENS'03, 
23 Jul. 2003, Banff, Alberta, Canada. IEEE, 2003, P. 103-107. DOI: 10.1109/ICMENS.2003.1221974.

25. Dey, Sukomal; Koul, Shiban K. "Reliability analysis of Ku-band 5-bit phase shifters using MEMS SP4T and SPDT switches," IEEE Trans. Microwave Theory Tech., Vol. 63, No. 12, P. 3997-4012, Dec. 2015. DOI: 10.1109/TMTT.2015.2491938.

26. Souchon, F.; Charvet, PL.; Maeder-Pachurka, C.; Audoin, M. "Dielectric charging sensitivity on MEMS switches," Proc. of Int. Conf. on Solid-State Sensors, Actuators and Microsystems, 10-14 Jun. 2007, Lyon, France. IEEE, 2007, P. 363-366. DOI: 10.1109/SENSOR. 2007.4300143 .

27. Орлов, А. В.; Кукушкин, А. Е.; Афиногенов, И. А. "Применение МЭМС технологий в практике разработки электростатических реле," Вестник новгородского государственного университета, Т. 1, № 75, C. 124-128, 2013. URI: http://www.novsu.ru/file/1092668. 